\title{
Navigated and Robotized Transcranial Magnetic Stimulation based on 3D Laser Scans
}

\author{
Lars Richter $^{1,2}$, Ralf Bruder ${ }^{1}$, Peter Trillenberg $^{3}$, Achim Schweikard ${ }^{1}$ \\ ${ }^{1}$ Institute for Robotics and Cognitive Systems, University of Lübeck \\ ${ }^{2}$ Graduate School for Computing in Medicine and Life Sciences, University of Lübeck \\ ${ }^{3}$ Departement for Neurology, University of Lübeck \\ richter@rob.uni-luebeck.de
}

\begin{abstract}
Navigated and robotized Transcranial Magnetic Stimulation (TMS) is advancing forward in research and treatment. MRI-scans or other medical image data are typically used as navigation source. Unfortunately, scanning time is always short and expensive. For many TMSapplications the underlying brain topology is not necessarily needed. Therefore, we generate the subject's head contour for a precise stimulation from 3D laser scans. We use the PowerCrust algorithm generating a smooth contour from laser surface points. The mean error found was 0.289 with an RMS error of $0.358 \mathrm{~mm}$ and a maximum error of $0.984 \mathrm{~mm}$ comparing MRI-scan to laser scan for a head phantom . Currently, we are working successfully with head contours based on 3D laser scans for two ongoing TMS studies. During these TMS-experiments, the overall error found for the robotized TMS system using laser scans was $<5 \mathrm{~mm}$. With new and faster laser technologies, it will be possible to use laser scans as navigation source and for direct head tracking during stimulation when scanner become real time capable.
\end{abstract}

\section{Introduction}

The aim of transcranial magnetic stimulation (TMS) is to stimulate the brain non-invasively and painlessly using a magnetic coil on top of the patient's head. Besides usage as a diagnostic tool, TMS is used as a treatment attempt in different neurological diseases, e.g. depression, chronic tinnitus or chronic pain [1]. For TMS, navigated stimulation has become state-of-the-art, at least in research, and is available in many TMS systems [2]. Mostly, (f)MRI-scans are used as navigation source. Unfortunately, MRI-scans are expansive and scanning time is often short. Robotized systems for TMS are advancing forward and are claimed more and more for exact stimulation [3]. For the robotized TMS system, a magnetic coil is placed directly on top of a patient's head by the robot being able to induce an electric current in a predefined region of the brain [4].

For treatment attempts of neurological disorders like tinnitus or schizophrenia, the stimulation point is selected mostly based on fMRI information. Moreover, for brain mapping purposes the underlying brain structure is essential for successful and meaningful investigations. For many other TMS applications the 
underlying brain topology is not necessarily required: For research applications and investigations, a hot spot search is performed finding an optimal stimulation point for each single subject by measuring the motor evoked potentials (MEPs) of a specific muscle. Underlying brain structure is not needed for this purpose [5]. Instead, it is more important to stimulate precisely at the hot spot and to reaccess the hot spot in different trials or days.

As MRI-scanner time is often hard to access and expensive, we propose usage of 3D laser scanner to obtain a precise three dimensional contour of the subject's head that can be used for navigated and robotized TMS. Compared to MRI scanner, laser scanner are easy to use and affordable. These systems are well established in medical applications. Previous investigations have shown that 3D laser scanner are suitable for 3D recordings of the human face [6]. Thus, 3D laser systems could be used to generate a complete 3D surface of the head. We evaluate the generated contour by laser scans by comparison with an MRI-scan and with manual head contour generation. Additionally, we present exemplarily the application in ongoing TMS investigations with the robotized system.

\section{Materials and Methods}

\subsection{Hardware Setup}

We use a GALAXY laser system (LAP GmbH Laser Applikationen, Lüneburg, Germany) for head scanning and a human head phantom for testing. The GALAXY laser scanner has a scan volume of $670-800 \times 950-1300 \times 490-$ $600 \mathrm{~mm}^{3}$. The scanning time depends on the resolution and on the size of scanning volume. The time needed to perform one scan is in the range of $1-5 \mathrm{~s}$. With a reduced resolution and in real time mode, the laser system can reach a scanning frequency up to $5 \mathrm{~Hz}$. The scanner has a repeatability of $<0.1 \mathrm{~mm}$ and an accuracy in the acquired patient position of $<1 \mathrm{~mm}$. The resolution in the measurement axis of the laser scanner is specified with $0.2 \mathrm{~mm}$ for the $\mathrm{y}$ and z-axis, and $0.5 \mathrm{~mm}$ for the $\mathrm{x}$-axis [7].

\subsection{Head Scanning \& Contour Generation}

A high resolution laser scan of a human head consists of $\approx 9000$ surface points. The resulting laser image is shown in Figure 1(a). In Figure 1(b) the single data points are visualized. A smooth head contour of single data points representing the head is generated with the PowerCrust algorithm [8]. Figure 1(d) shows the generated contour of the head phantom. In Figure 1(c) the contour is illustrated with the underlying data points. Hair is a critical issue as hair absorbs laser light. Therefore, we use white swimming caps that are tight-fitting to the head. Note that this cap is only needed for laser scan acquisition. In contrast, MRI head contour is generated using edge detection extracting the head surface in the MRI images. 


\section{Results}

\subsection{Comparison to MRI Scans}

We used a head phantom to compare head contour generated by 3D laser scans with head contour generated from MRI-scan. Therefore, we estimated the distance from every data point of laser scan to MRI head contour. Ten different head phantom positions and scans were used. The mean error found was $0.289 \mathrm{~mm}$ with an RMS error of $0.358 \mathrm{~mm}$ and a maximum error of $0.984 \mathrm{~mm}$. Roughly, $10-15 \%$ of data points had to be excluded due to noise and deflections, having still $6000-8000$ data points for matching. Figure 2a illustrates a laser scan overlaying the MRI head contour.

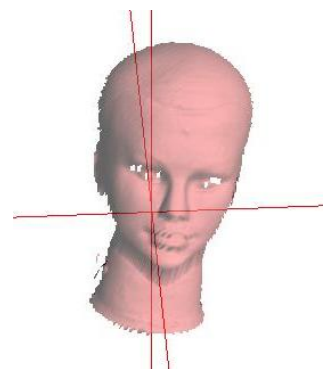

(a)

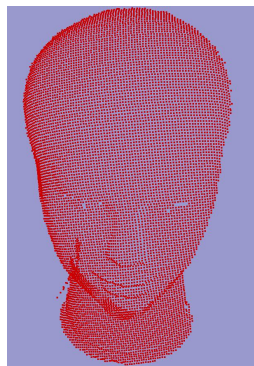

(b)

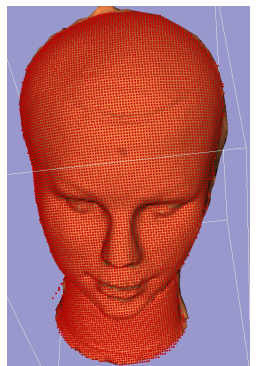

(c)

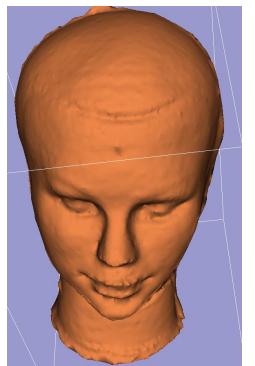

(d)

Fig. 1. Head phantom scanned by laser scanner. (a) Resulting scan in the scanning software. (b) Each scan consists of $\approx 9000$ single data points. (c) From the single data points a head contour is computed. (d) Head contour computed with the PowerCrust method [8].

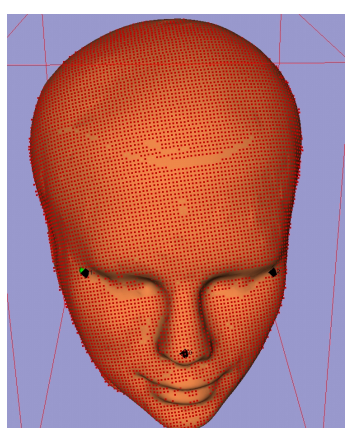

(a)

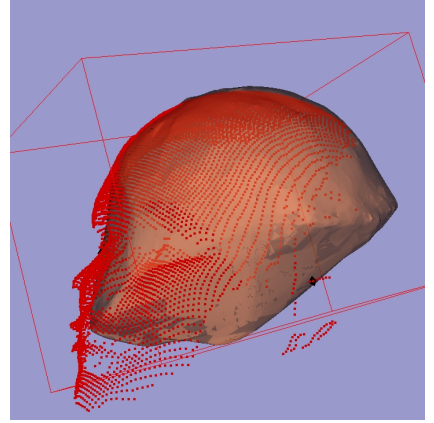

(b)

Fig. 2. MRI head contour of head phantom with overlying 3D laser scan. (a) larger dark dots mark initial landmarks for registration; (b) manually generated. 


\subsection{Comparison to Manual Contour Generation}

A Pointer tracked by a Polaris Spectra stereo optic infrared tracking camera (Northern Digital Inc., Waterloo, Ontario, Canada) can be used to generate a manual head contour. For this purpose, the pointer is continuously tracked while moved on the head surface. Typically, with this method 500-1000 surface points are collected. Using again the PowerCrust algorithm [8] a head contour can be generated. Figure $2 \mathrm{~b}$ visualizes the inexactness of manual head generation compared to 3D laser scans.

\subsection{Usage in TMS Studies}

Currently, we are successfully using head contours based on 3D laser scans for two ongoing TMS studies with 20 subjects, so far.For both the studies, motor cortex mapping has to be performed. A stimulation hot spot for right foot and for left hand are identified for the first and second experiment, respectively. Once the hot spot is found, the coil will be positioned exactly at the hot spot again for stimulation. Figure 3 shows motor cortex mapping results for two subjects.

During these TMS-experiments, we found that the overall error of the robotized system using laser scans is $<5 \mathrm{~mm}$. This was measured as the maximum distance between coil and head. Note that robot-tracking calibration, coil and head registration, and hair influence the accuracy besides the laser scan.

\section{Discussion}

We have shown that 3D laser scans of the head can be used as a navigation source for TMS when no medical image data is on hand. Instead of a manual head contour generation, where the data is collected with a pointer, it is more
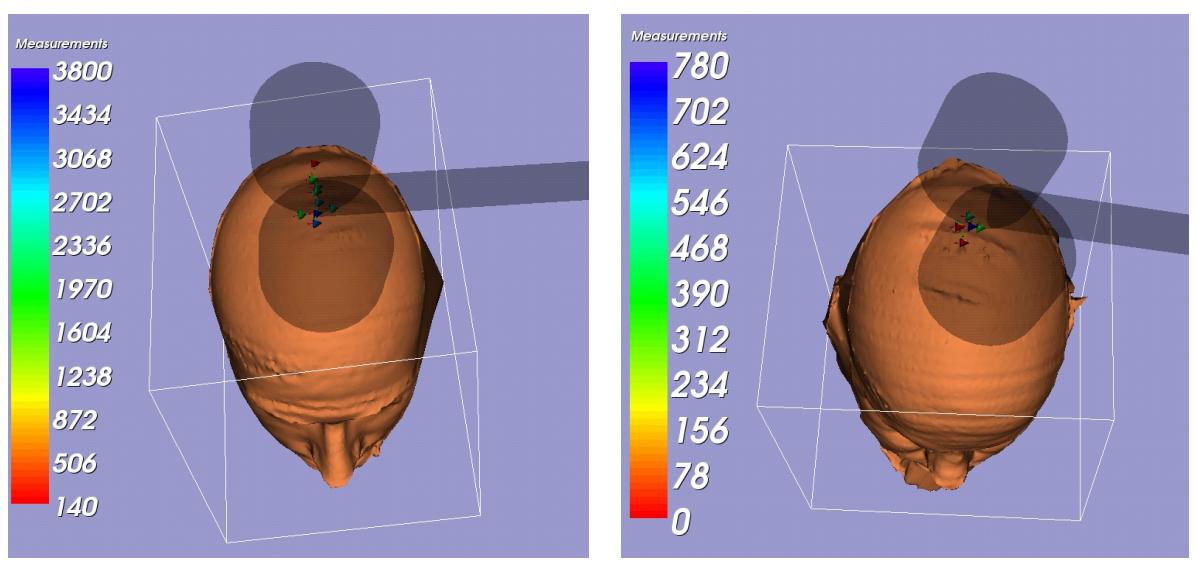

Fig. 3. Motor cortex mapping results based on laser scans. 
precise and appropriate to the head due to the fact that the laser scan consists of a magnitude more points compared to a manual head contour.

Our ongoing clinical trials have practically proven that 3D laser scans are sufficient for application in navigated and robotized TMS systems. For a later evaluation, registration of laser scans with acquired measurements to medical images is possible.

When data acquisition of 3D laser scans becomes real-time capable with new technologies, it will be possible to use laser scans as navigation source and for direct head tracking during stimulation [9]. This would speed up the whole process and increase the acceptance of the system in clinical workflow as subjects could be stimulated without any data preparation or registration.

Acknowledgement. The authors would like to thank LAP GmbH Laser Applikationen for providing the 3D laser system and equipment. This work was partially supported by the Graduate School for Computing in Medicine and Life Sciences funded by Germany's Excellence Initiative [DFG GSC 235/1].

\section{References}

1. Pascual-Leone A, Davey NJ, Rothwell J, et al. Handbook of Transcranial Magnetic Stimulation. Arnold; 2002.

2. Ruohonen J, Karhu J. Navigated transcranial magnetic stimulation. Clin Neurophysiol. 2010;40(1):7-17.

3. Langguth B, Kleinjung T, Landgrebe M, et al. rTMS for the treatment of tinnitus: the role of neuronavigation for coil positioning. Clin Neurophysiol. 2010;40(1):45-58.

4. Matthäus L, Trillenberg P, Bodensteiner C, et al. Robotized TMS for motion compensated navigated brain stimulation. In: Proc CARS; 2006. p. 373-8.

5. Balslev D, Braet W, McAllister C, et al. Inter-individual variability in optimal current direction for transcranial magnetic stimulation of the motor cortex. J Neurosci Methods. 2007;162(1-2):309 - 13.

6. Kovacs L, Zimmermann A, Brockmann G, et al. Three-dimensional recording of the human face with a 3D laser scanner. J Plast Reconstr Aesthet Surg. 2006;59(11):1193 -202 .

7. LAP-Laser. GALAXY: Patient Topography Laser System. Tech Rep. 2009; p. $245-61$.

8. Amenta N, Choi S, Kolluri R. The power crust, unions of balls, and the medial axis transform. Comput Geometry: Theory Appl. 2001;19:127-53.

9. Richter L, Bruder R, Schlaefer A, et al. Towards direct head navigation for robotguided transcranial magnetic stimulation using 3D laserscans: idea, Setup and feasibility. Proc IEEE EMBS. 2010; p. 2283-6. 